\title{
Extraction and Pre-concentration of Phthalic Acid Esters from Aqueous Solutions using Agitation-assisted Dispersed Binary Solvents Microextraction
}

\author{
Mosotho J. George ${ }^{a, c, \star}$ Ontiretse R. Tagwa ${ }^{b}$ and Kwenga F. Sichilongo ${ }^{c}$ \\ ${ }^{1}$ Department of Chemistry and Chemical Technology, National University of Lesotho, P.O. Roma 180, Lesotho. \\ ${ }^{2}$ Department of Biological Sciences, University of Botswana, Private Bag 0022, Gaborone, Botswana. \\ ${ }^{3}$ Department of Chemistry, University of Botswana, Private Bag 0022, Gaborone, Botswana. \\ Received 14 September 2016, revised 3 June 2017, accepted 17 July 2017.
}

\begin{abstract}
As the search for efficient, affordable and environmentally friendly extraction methods continues, miniaturized solvent techniques are receiving considerable attention due to their simplicity, efficiency and fast extraction rates. Herein we report application of a modified dispersive liquid-liquid microextraction through the introduction of a second extracting solvent in place of a disperser solvent leading to a much improved organic solvent recovery after the extraction of phthalic acid esters from aqueous solutions. The method employs a $75 \mu \mathrm{L}$ binary mixture of ethylacetate and tetrachloromethane with a 3:1 ratio, extraction time of 20 seconds, and $10 \% \mathrm{NaCl}$. The method demonstrates sufficient linearity $\left(R^{2} \geq 0.9866\right)$ and repeatability $(\% R S D \leq 12.5$, for $n=15)$ with the estimated LOD in the range $\left(0.01-0.05 \mathrm{ng} \mathrm{mL}^{-1}\right)$ using the $\mathrm{S} / \mathrm{N}$ approach. The method demonstrated significant enrichment factors averaging about 2100 with headspace and about 3000 and increase of over $40 \%$ without headspace, indicating the importance of reduced headspace volume in this approach, especially if the ionic strength is increased. However, the method yielded a significantly wide range of recoveries (65-115\%) when applied to the analysis of spiked soil samples when coupled to hot-water extraction. However, this may not be entirely attributable to the extraction approach but rather heat-induced hydrolysis during the hot-water dissolution, prior to the proposed organic extraction method.
\end{abstract}

KEYWORDS

Dispersive liquid-liquid microextraction, binary solvents, phthalic acid esters, headspace volume, extracting solvent recovery.

\section{Introduction}

Phthalic acid esters (PAEs), sometimes called phthalates, are some of the most ubiquitous plasticizers in use today with their application estimated at around $70 \%$ of the total world plasticizers consumption in 2014, ${ }^{1}$ and the projected total production of about 8 million tons. ${ }^{2}$ Their use is mainly in the softening of the hard plastic for different applications. Owing to their use in some domestic appliances, their leaching into the food or any liquid material they get into contact with cannot be overruled. In fact, they have been reported in various systems including: baby toys, baby foods, hospital tubes, not to mention the environment where they are most ubiquitous. ${ }^{3}$ Despite not showing considerable toxicity when exposed to laboratory animals, phthalates reportedly showed some endocrine disrupting and carcinogenic activity in some laboratory animals ${ }^{4}$ hence a call for their regulation by the US Food and Drug Authority. ${ }^{5}$ Consequently, these chemicals are receiving considerable attention from the public health practitioners leading to some manufacturers reportedly phasing out the processes that involve the use of these chemicals. ${ }^{6,7}$ Several recommendations exist for their maximum allowable levels in different regions. For example, the total tolerable daily intake of general PAEs has been capped at $0.3 \mathrm{mg} \mathrm{kg}^{-1}$ body in Europe while for individual PAEs this value is lowered to $0.1 \mathrm{mg} \mathrm{kg}^{-1}$ of body weight. ${ }^{8}$ The US EPA has set the limit for bis(2-ethylhexyl) phthalate commonly abbreviated as DHEP, the

* To whom correspondence should be addressed. E-mail.jm.george@nul.ls / maluti2005@gmail.com most common PAE, at the $6 \mathrm{ng} \mathrm{mL} \mathrm{m}^{-1}$ in drinking water. ${ }^{9}$ A very well presented review of the socio-economic issues involved in the control of the PAEs was reported in 2003..$^{10}$

As most other organic compounds, PAEs can be determined using a range of techniques from electrochemical to spectroscopy-based. To this effect different detection approaches have been reported for the PAEs analyses, namely, spectroscopy using either molecular absorption in the form of infrared, ${ }^{11}$ ultraviolet-visible spectrophotometry, ${ }^{12}$ spectrofluorimetry, ${ }_{1}^{13}$ or electrochemistry. ${ }^{14}$ However, chromatography is still a method of choice owing to its ability not only to detect but also to separate the innate complex matrices that these compounds exist in in nature since chromatography separates them firstly into individual compounds prior to detection using any detection methods.

However, despite its applicability, chromatography still requires prior sample treatment to render the analytes analysable in terms of concentration range, structural forms and even reduction of the matric complexity since these can also bring about more complications in the process. As such extensive research is focused on developing effective, quick and yet affordable methods for determination of organic chemicals such as PAEs and other compounds of interest. Solvent extraction is still the only method used for extraction of organic compounds from solid matrices with a range of approaches such as Soxhlet extraction, ${ }^{15}$ sonication, ${ }^{16}$ pressurized liquid, ${ }^{17}$ supercritical ${ }_{,}^{18}$ microwave-assisted, ${ }^{19}$ employing either organic or aqueous sol- 
vents for the dissolution. Recently a simple reflux was compared and faired comparatively with Soxhlet extraction for extraction of PAEs from the solid-waste dumpsite soil. ${ }^{20}$

Due to concerns of the use and disposal of copious organic solvents that are by themselves of environmental concern, miniaturization seems an obvious choice. Consequently, liquid-based miniaturized approaches are receiving considerable attention judging by the number of articles that have been published since the first report of the sampling in a drop in the mid-1990s. ${ }^{21,22}$ This approach has seen a number of different formats each of which reports some degree of success and benefit in some of the figures of merits that are often considered, most notably the limits of detection (LODs), speed of method, cost and ease of handling to mention but a few attributes as reviewed recently. ${ }^{23}$ Different variants include: drop-based, supported liquid and dispersed solvent microextraction each demonstrating certain benefits over others. For example, supported liquid microextraction can be used as passive samplers while the other two cannot, dispersive solvent microextraction is the most rapid while it requires a lot more solvent and leads to poorer solvent recovery than the drop-based solvent microextraction. Some of the modifications are as simple as an introduction of a mere air-bubble in the drop to enable enhanced dynamic extraction ${ }^{24}$ or an increased surface area to volume ratio as well as the increased buoyancy of the droplet in the case of denser solvents being used, ${ }^{25}$ all of which enhance extraction efficiency.

Recently, there have been reports on modifying DLLME and coupling it to solid-phase microextraction ${ }^{26}$ and hollow-fibre liquid-liquid micro extraction ${ }^{27}$ despite the former being accompanied by concomitant fibre swelling. This manuscript presents an attempt to employ a modified binary-liquid mixture dispersive liquid-liquid microextraction by replacing a dispersing liquid for a second extracting solvent in an effort to improve the reported poor solvent recovery post-extraction. ${ }^{28}$ In addition to the improved solvent recovery, the mixed-solvent extraction has been reported to improve extraction efficiency that any of the individual pure solvents used in a mixture. ${ }^{29,30}$ In the current report, combination of tetrachloromethane with ethylacetate demonstrated the highest efficiency. Otherwise, most attributes closely resemble the normal DLLME except the reported excessive solvent loss associated with classical DLLME.

\section{Experimental}

\subsection{Chemicals and Reagents}

A phthalate esters mixture (Mix 3: P.I. 08060300) containing benzylbenzoate and the following phthalic acid esters: dimethyl-, diethyl-, diisobutyl-, dibutyl-, dimethylglycol-, bis(4-methyl-2-pentyl)-, bisethoxyethyl-, bispentyl-, hexyl-2ethylhexyl-, benzylbutyl-, bis-hexyl-, bis-n-butoxyethyl-, biscyclohexyl-, bis-2ethylhexyl-, bisoctyl, bisnonyl- and bis(7-methyloctyl)- respectively at 1000 ppm each was obtained from Dr Ehrenstorfer GmbH (Augsburg, Germany) in a $100 \mathrm{~mL}$ ampule. All solvents used in this study were HPLC or GC grade: methanol, toluene, chloroform, dichloromethane, tetrachloromethane, hexane, toluene and water were all obtained from Riedel-de Haën (Steinheim Germany). Ethylacetate (AR) was obtained from Associated Chemicals Enterprises (Pty) Ltd (South Africa).

\subsection{Instrumental Methods}

A 7890A Agilent GC-MS fitted with a J\&W HP-5MS column with $30 \mathrm{~m} \times 0.25 \mathrm{~cm} \times 0.25 \mu \mathrm{m}$ dimensions was used. The chromatographic method was developed as follows: injection port was set at $280{ }^{\circ} \mathrm{C}$; column program: initial temperature of $80^{\circ} \mathrm{C}$ for $4 \mathrm{~min}$, then ramped at $10^{\circ} \mathrm{C} \mathrm{min}^{-1}$ to $300^{\circ} \mathrm{C}$ and held for 5 min; the transfer line was set at $280^{\circ} \mathrm{C}$. The carrier gas was UHP Helium at a flow rate of $1 \mathrm{~mL} \mathrm{~min}^{-1}$ throughout the analyses. The autoinjector was programmed to inject $2 \mu \mathrm{L}$ with rinsing before and after the injections. The MS was set on scanning mode, scan range of 50-500 atomic mass units, for identification of samples; otherwise for quantitative analyses it was set on SIM mode using the respective mass spectra base peakions of the individual compounds as well as their qualifying ions (see Table 1 ). The temperature settings were $230{ }^{\circ} \mathrm{C}$ for the ion source and $150{ }^{\circ} \mathrm{C}$ for the quadrupole.

\subsection{Development and Optimization of the Extraction Protocol}

Different aliquots of appropriate volumes containing the phthalate mixture $\left(1 \mu \mathrm{g} \mathrm{mL}^{-1}\right)$ were prepared in HPLC grade water. Appropriate volumes of the organic solvents were added appropriately and shaken for a given time. Thereafter the solu-

Table 1 The phthalic acid esters and their respective chromatography and mass spectral data obtained in this study.

\begin{tabular}{|c|c|c|c|c|c|}
\hline Compound & $\begin{array}{l}\text { Time } \\
/ \mathrm{min}\end{array}$ & $\begin{array}{l}\text { Molecular mass } \\
\text { /amu }\end{array}$ & Reference ion* & Qualifying ion & SIM/Qual ratio \\
\hline Dimethylphthalate & 12.957 & 194 & 163 & 77 & 5.342 \\
\hline Benzylbenzoate & 16.685 & 212 & 105 & 212 & 3.330 \\
\hline Diethylphthalate & 17.702 & 222 & 149 & 177 & 3.902 \\
\hline Diisobutylphthalate & 17.705 & 278 & 149 & 223 & 11.352 \\
\hline Dibutylphthalate & 18.643 & 278 & 149 & 223 & 16.377 \\
\hline Dimethylglycolphthalate & 19.608 & 292 & 59 & 149 & 3.364 \\
\hline Bis(4-methyl-2-pentyl)phthalate & 19.775 & 334 & 149 & 85 & 2.425 \\
\hline Bisethoxyethylphthalate & 20.125 & 310 & 72 & 149 & 1.224 \\
\hline Bispentylphthtalate & 20.438 & 306 & 149 & 237 & 11.893 \\
\hline Hexyl-2-ethylhexylphthalate & 22.090 & 334 & 149 & 251 & 10.742 \\
\hline Benzylbutylphthalate & 22.221 & 312 & 149 & 206 & 3.568 \\
\hline Bis-hexylphthalate & 22.897 & 362 & 149 & 251 & 7.866 \\
\hline Bis-nbutoxyethylphthalate & 23.147 & 366 & 149 & 193 & 2.871 \\
\hline Biscyclohexylphthalate & 23.578 & 330 & 149 & 167 & 2.938 \\
\hline Bis-2ethylhexylphthalate & 23.672 & 390 & 149 & 167 & 2.783 \\
\hline Bisoctylphthalate & 25.061 & 390 & 149 & 279 & 9.000 \\
\hline Bisnonylphthalate & 25.164 & 418 & 149 & 293 & 3.842 \\
\hline Bis(7-methyloctyl) phthalate & 25.643 & 418 & 149 & 293 & 3.481 \\
\hline
\end{tabular}

* This is the ion that represents the base peak and used in selected ion monitoring. 
tions were centrifuged to sediment the heavier organic solvent. The sedimented organic solvent was transferred to a GC-vial fitted with a glass-insert with the capacity of $100 \mu \mathrm{L}$, thereafter the vials were loaded to the autosampler for onward injection and analysis. All the analyses were run in triplicate $(n=3)$ except where it is expressly stated otherwise.

A univariate approach was followed to determine optimum conditions for the following: solvent choice, mixed-solvent, solvent volume, extraction time, ionic strength as well as the solution volume. Thereafter the extraction efficiency of the method was determined combining all the optimized conditions (factors) appropriately. Details of each procedure will be provided under relevant section of the results and discussions, where it differed from the outlined procedure.

\subsection{Application of the Extraction Method to the Real Soil Samples}

To investigate the applicability of the method for the determination of the PAEs from soil samples, a soil sample obtained from a solid-waste dumpsite sample, was used for the determination of the esters in the soil sample coupled to hot-water extraction described elsewhere. ${ }^{31}$ Thereafter, $5 \mathrm{~g}$ sample portions of this soil were extracted using hot-water extraction using $5 \mathrm{~mL}$ of hot water to achieve a $1 \mathrm{~g} \mathrm{~mL}^{-1}$ suspension. The suspension was centrifuged appropriately and the supernatant extract was subjected to the developed method at room temperature in order to determine the amount of the PAEs in the soil.

To determine the degree of analyte recovery from the soil sample, the soil sample was pre-extracted with Soxhlet extraction remove all the PAEs from the dumpsite. Thereafter, this soil was air-dried overnight in an oven set at $40{ }^{\circ} \mathrm{C}$. A portion of $19 \mathrm{~g}$ of the dried pre-extracted soil was spiked with $1.9 \mathrm{~mL}$ of $10 \mu \mathrm{g}$ $\mathrm{mL}^{-1}$ aqueous solution to achieve a $1 \mu \mathrm{g} \mathrm{mL}^{-1}$ spiked solution following the report of traceless spiking using aqueous as opposed to the organic standards. ${ }^{22,32}$ The soil was submerged under water to enable even distribution of the analytes. The soil was allowed to age naturally by being placed on the windowsill until all the water had evaporated. Thereafter, a further 21 days was allowed to age the soil sample. Subsequently the same procedure was followed as for the parent sample in the preceding paragraph.

\section{Results and Discussion}

\subsection{Optimization of the Separation of the Mixtures}

Following the injection of the standard mixture, some chromatographic and mass spectrometry analyses were made to identify and characterize the peaks with respect to the analytes listed on the information for the standards package. Table 1 shows the analytes and their respective chromatographic and mass spectral properties obtained in this study: retention time, relative molecular mass, the base peak (used as reference peak for selected ion monitoring - SIM), the qualifying ion (second in abundance) and the ratios of the intensities of SIM ion to qualifying ion (Ref/Qual) in the mass spectra to be used in the identification of these compounds from the real water samples.

As can be seen from Table 1, with the exception of dimethylphthalate, benzylbenzoate, dimethylglycolphthalate and bisethoxyethylphthalate, all the esters show the base peak at 149 which is characteristic of the phthalic acid esters due to some rearrangement during the mass spectral analysis as discussed elsewhere in literature. ${ }^{33}$

\subsection{Determination of the Ideal Solvent for the Extraction}

From the basic requirement of the sedimentation-based DLLME method, density of the solvent is very important; as such only chlorinated solvents are applicable as they are denser than water so they can settle at the bottom of the aqueous solution after shaking. However chlorinated solvents are already an environmental and health hazard as such only a few were tested. From a previous report ${ }^{17}$ it was established that the PAEs extracted better in ethylacetate. From the polarity of ethylacetate (4.4) compared to that of the chlorinated solvents, chloroform affords the closest (4.1). So, the preliminary experiment was performed to assess the combination of chloroform with ethylacetate since ethylacetate on its own would not afford the properties anticipated - sedimenting after the extraction. Thus different combinations of chloroform-ethylacetate were tested for their respective efficiencies starting with $100 \%$ chloroform and increasing the percentage of ethylacetate. Figure 1 shows the effect of adding the ethylacetate into the chloroform on the extractability of the esters with the results calculated relative to $100 \%$ chloroform. Only ten PAEs are presented in this figure for

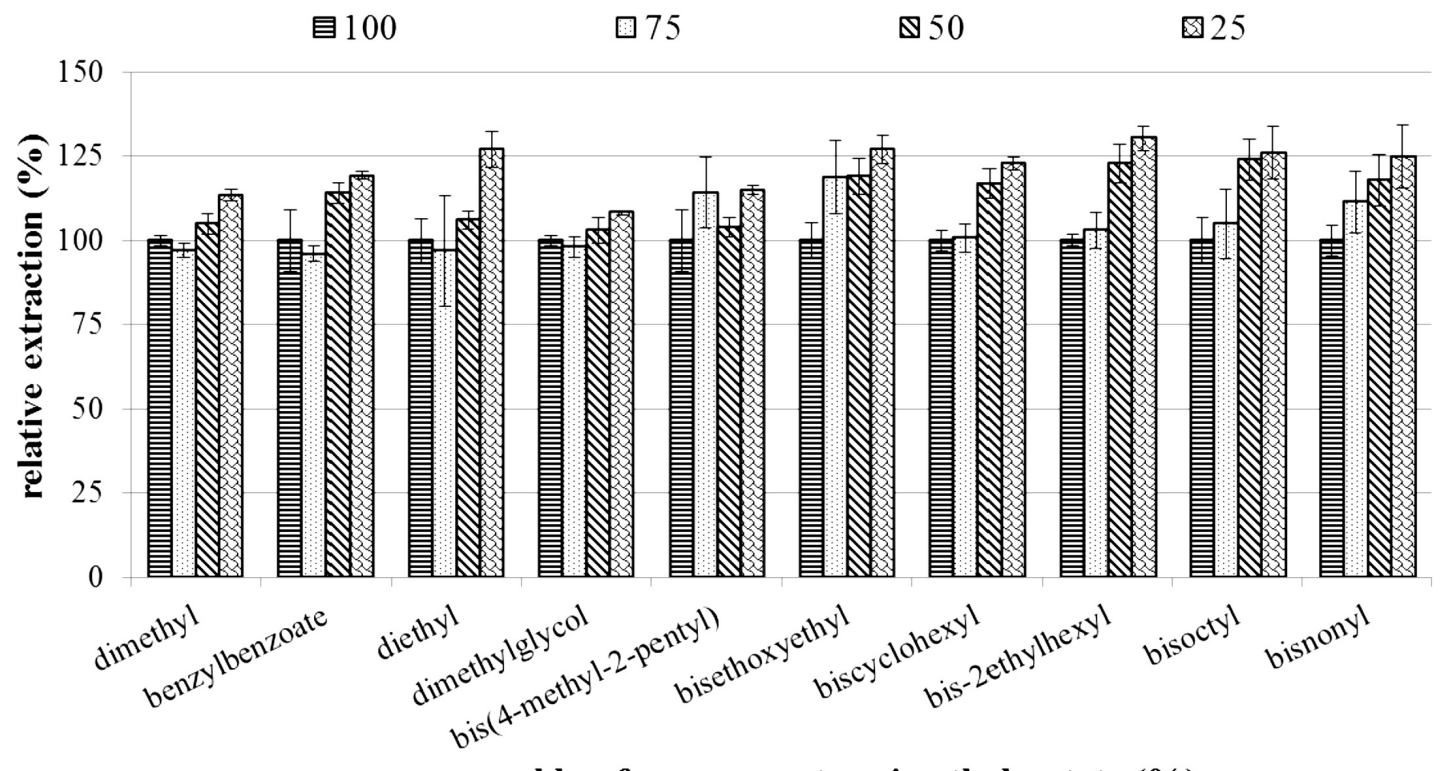

chloroform percentage in ethylacetate $(\%)$

Figure 1 Effect of different chloroform-ethyl acetate mixtures on the extractability of the PAEs. 
clarity of presentation, and these were picked randomly from the 18 compounds appearing in Table 1.

As can be seen, the extraction efficiency increased with the increase in ethyl acetate percentage. This was however accompanied by significant solvent loss: $100 \%$ chloroform ( $\pm 0 \%$ loss), $75 \%$ chloroform ( $\pm 10 \%$ loss), $50 \%$ chloroform ( $\pm 25 \%$ loss) and $25 \%$ chloroform ( $\pm 50 \%$ loss).

In order to reduce the solvent loss a less polar solvent was thought about, hence the other chlorinated solvents were tried in combination with ethyl acetate (see Fig. 2). The results are plotted relative to chloroform mixture with the ethyl acetate (3:1 ratio as determined before) with the error bars indicating the relative standard deviations of the triplicate analyses.

Clearly ethylacetate:tetrachloromethane mixture affords better extraction efficiency than chloroform and dichloromethane mixtures averaging just over $200 \%$ of chloroform while dichloromethane averaged about $120 \%$ of chloroform efficiency. This could be attributed to the lower combined polarity since the polarity of tetrachloromethane is 1.6 , compared to 4.1 of chloroform. Thus the combination of ethylacetate with tetrachloromethane (3:1) was used for further experiments. The observed solvent loss was slightly reduced (about 60-70 \% was recovered at $75 \%$ ethylacetate) in this case of using tetrachloromethane.

\subsection{The Effect of Varying the Volume of the \\ Extracting Solvent}

Different volumes (50-200 $\mu \mathrm{L})$ of 3:1 ethylacetate:tetrachloromethane were used and their extraction compared accordingly. The results presented in Fig. 3 are for the 10 compounds that gave results with more difference between the different volumes. These results were still accompanied by severe solvent loss averaging at best $40 \%$, considering the volumes were not measured accurately since the minimum calibration of the pipette used was $10 \mu \mathrm{L}$, so the recovered volumes were rounded to the nearest $10 \mu \mathrm{L}$.

Clearly from Fig. 3, the increase in solvent volume decreases extraction efficiency, this is possibly due to dilution. However, from 75 to $50 \mu \mathrm{L}$, there is a slight increase for some compounds, while others show a drop in extraction. This could be attributed to the severe reduction in volume of the extracting solvent, making it difficult to withdraw only the organic solvent from the solutions. The volume of $50 \mu \mathrm{L}$ was accompanied by relatively higher deviations averaging $10 \%$ compared to just over $5 \%$

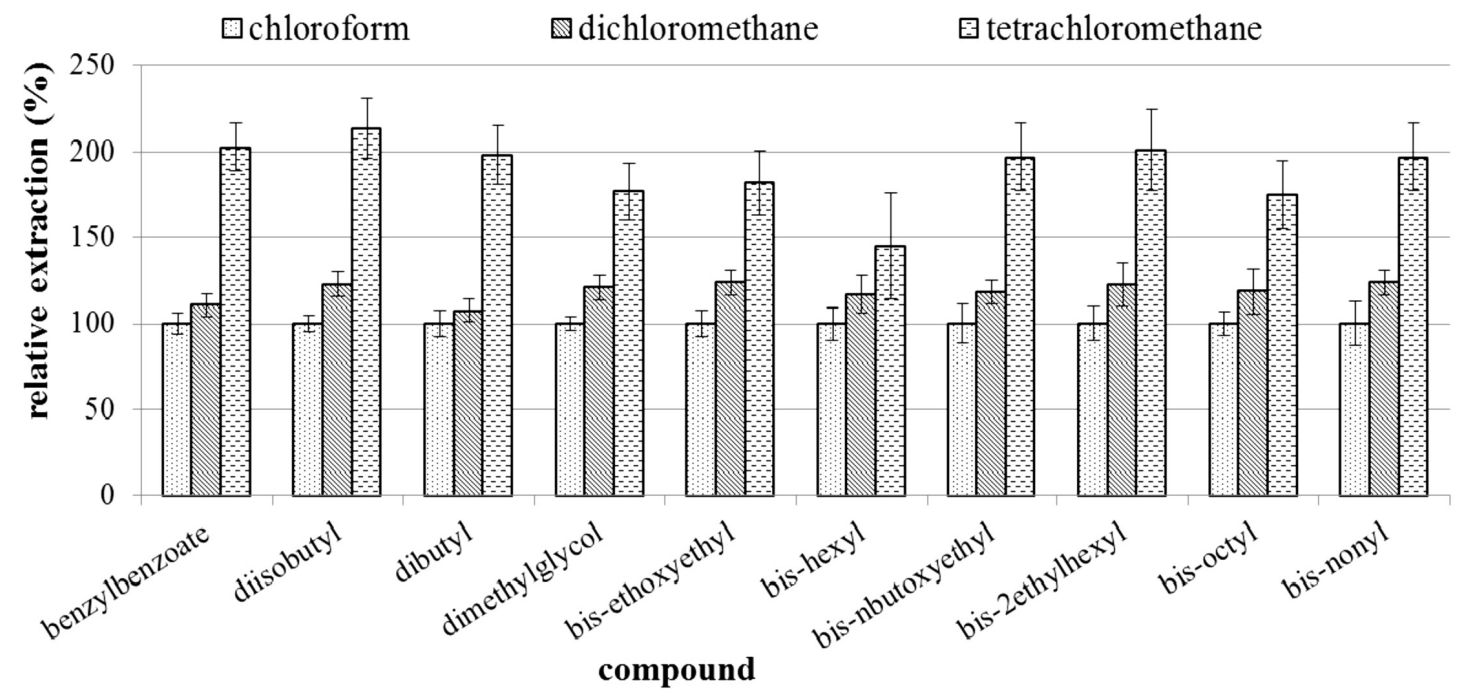

Figure 2 Extractability of the different PAEs in different chlorinated solvents relative to chloroform (only few compounds were selected randomly for clarity of the presentation).

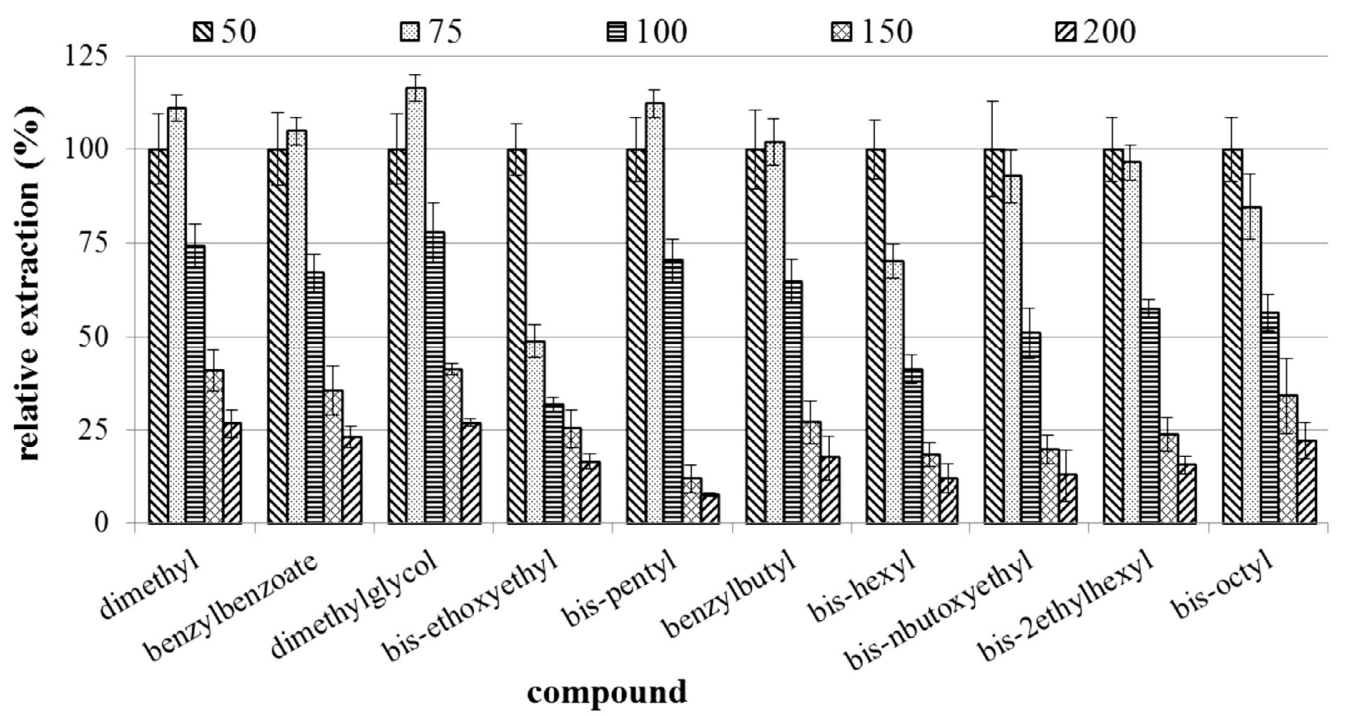

Figure 3 Effect of the increase in extracting solvent volume on the efficiency. 
obtained with $75 \mu \mathrm{L}$ volume. A similar behaviour has reported while using a simple solvent extraction of the soil sample using organic solvents. ${ }^{17}$ Thus a volume of $75 \mu \mathrm{L}$ was adopted as the working volume for further experiments.

\subsection{Effect of Ionic Strength}

Different amounts of sodium chloride $(0.025,0.05,0.075,0.1$, 0.15 and 0.2 ) were weighed and added to different $1-\mathrm{mL}$ aqueous solutions. Thereafter the solutions were extracted as usual. Figure 4 shows the effect of increasing ionic strength on the extraction relative to the extractability without the added sodium chloride $(\% \mathrm{NaCl})$. Only ten analytes were randomly picked for clarity of presentation.

There seems to be a slight increase in extraction efficiency with the increase in sodium chloride and peaking at about $10 \%$ for most compounds with an average increase of about $20 \%$ compared to $0 \% \mathrm{NaCl}$. There is a drop in extraction efficiency from $15 \%$ onwards attributable to a well-known salting out effect. However, given that there were two organic solvents used, the question was whether the salting out effect only affected the analytes and not the solvents, in which case the reported efficiency could be spurious. To investigate this further the effect of headspace was determined.

\subsection{Effect of Headspace Volume on the Extraction Efficiency}

The effect of headspace volume was investigated using only two modes, with or without headspace and in the presence of $10 \% \mathrm{NaCl}$. In measurement with the headspace volume, the usual procedure was followed, $1 \mathrm{~mL}$ of the sample and $75 \mu \mathrm{L}$ of the solvent, while that without the headspace volume, the solution was topped-up after the addition of the extraction solvent mixtures while maintaining the ratio of the aqueous solution to the extracting solvent $(1 \mathrm{~mL}$ to $75 \mu \mathrm{L})$. After the equilibration the volume of the recovered solution was measured and $2 \mu \mathrm{L}$ of which was injected into the GC-MS for analysis.

The results showed an average improvement of about $157 \%$ in the extraction efficiency in the absence of headspace (see Table 2). A closer look at the internal standard response also revealed the recovery improvement of about $25 \%$ without the headspace. This, therefore, suggests that the added sodium chloride does not only decrease the extraction efficiency, but does so through the salting out of the organic solvent into the headspace, hence a reduction in efficiency of extraction. This was in agreement with the reported literature that addition of
$\mathrm{NaCl}$ beyond a certain threshold drives the organic solvent out of the solution as evidenced by the increased volume of organic solvent on the surface of the aqueous solution. ${ }^{34}$ On the other hand, the issue of the internal standard leaching into the aqueous solution and its reduction in the presence of salts in solutions was reported elsewhere in the work involving single-drop microextraction. ${ }^{35}$ Therefore, a minimum to no headspace volume was maintained for the subsequent experiments. It is worth mentioning that although no headspace was necessary, it became difficult to add the organic solvent and disperse it thoroughly without any headspace volume, hence only a minimum volume was left for the subsequent experiments.

\subsection{Effect of Varying the Extraction Time on the} Extraction Efficiency

Typically, DLLME is characterized by quick extraction times. To determine the optimum time, a series of solutions were prepared and extracted at different times, then the sediment was injected into the gas chromatograph and the extraction efficiencies compared as in Fig. 5.

As can be seen, the extraction is almost insensitive to time variation with only a small increase (less than $5 \%$ on average) from 20 seconds and 30 seconds. Therefore, 20 seconds was chosen as an ideal extraction time to avoid fatigue since the agitation was carried out using a simple manual vortex mixer.

\subsection{Validation of the Method and Comparison of its}

Performance with the Literature

The optimized conditions for the method can be summarized as follows: $75 \mu \mathrm{L}$ of 3:1 ethylacetate:tetrachloromethane solvent mixture, no-to-minimal headspace allowed, $10 \% \mathrm{NaCl}$ with 20 seconds agitation time. The effect of $\mathrm{pH}$ was not investigated as it is generally known that esters hydrolyse in both acidic ${ }^{36}$ and basic media. ${ }^{37}$ The method optimized method was validated for reproducibility and repeatability using different spiking levels of the phthalates and extracted them a minimum of $n=15$ (5 solutions over 3 days). The results demonstrated very good reproducibility with the \%RD ranging between 3 and 7.5 with the internal standard and 7 and 12.8 without the internal standard. Table 2 summarizes some of the analytical parameters determined herein including the enrichment factors of the analytes obtained under optimum conditions as determined.

As can be seen, the method yielded sufficient linearity with the coefficient of determination, $R^{2}$ in the range 0.9866 and 0.9993 in

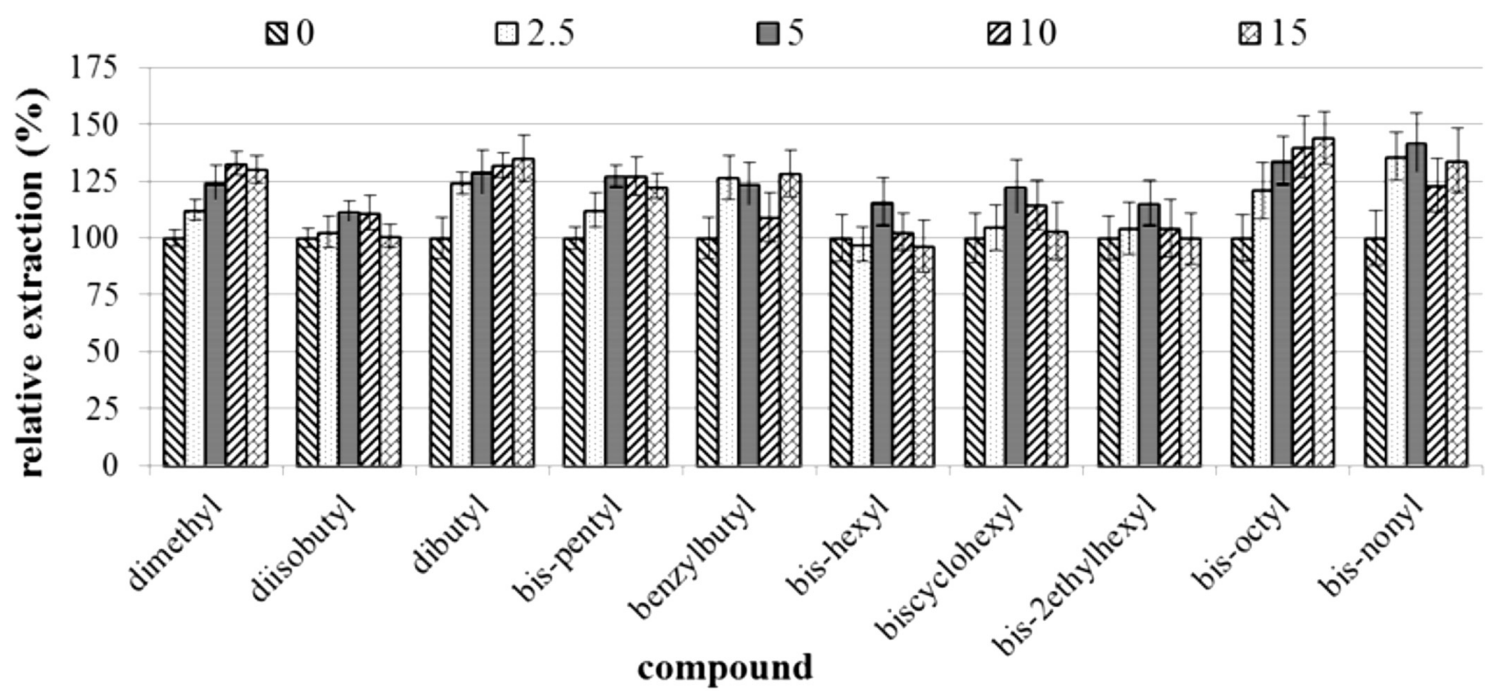

Figure 4 Effect of varying sodium chloride amount (\%) on the extractability of the PAEs . 


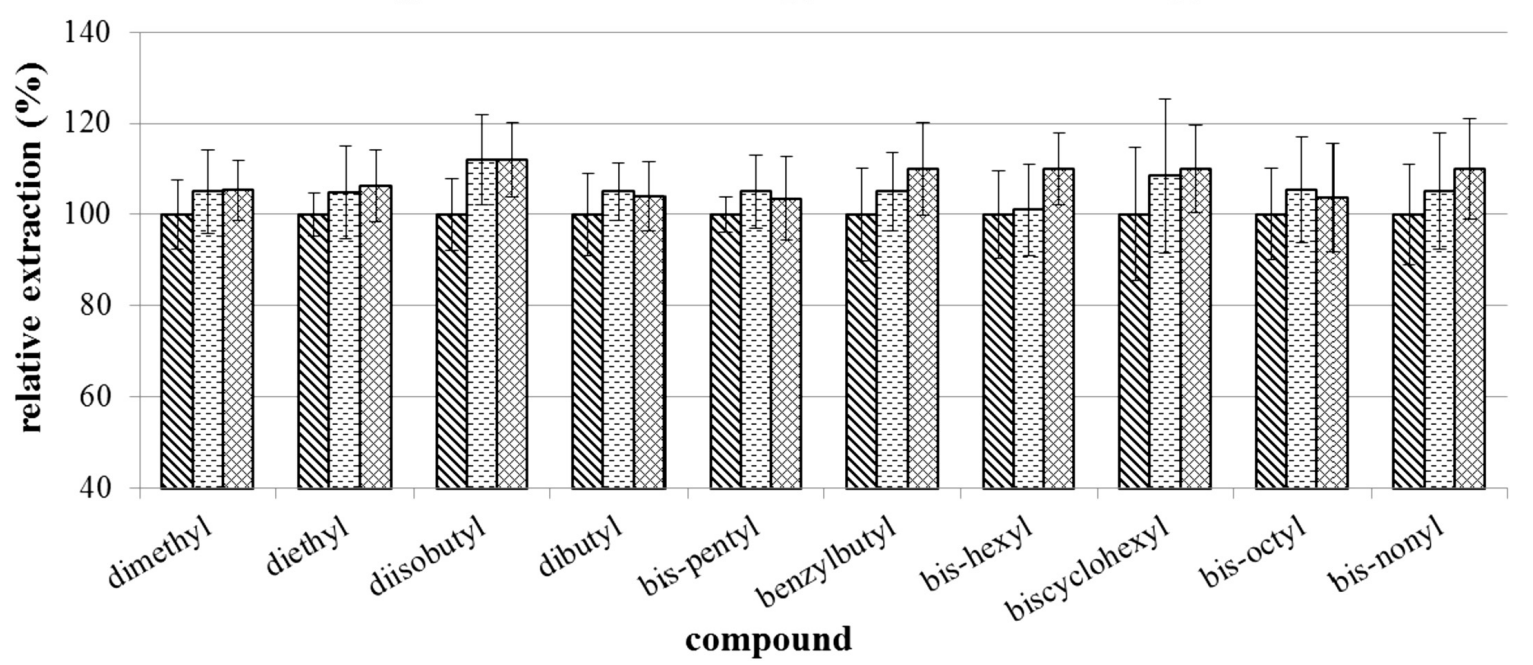

Figure 5 Effect of varying the agitation time (in seconds) on the extraction efficiency.

Table 2 Some analytical data obtained from the optimized conditions: enrichment factors with and without the headspace volume, linearity, the regression equation and the estimated limit of detection for each ester.

\begin{tabular}{|c|c|c|c|c|c|}
\hline & $\begin{array}{l}\text { Enrichment without } \\
\text { headspace }\end{array}$ & $\begin{array}{l}\text { Enrich. Ratio (with/without } \\
\text { headspace) * }\end{array}$ & $\begin{array}{l}\text { Repeatability } \\
\text { /\% RSD }\end{array}$ & $\underset{R^{2}}{\text { Linearity }}$ & $\begin{array}{l}\text { Est. LOD } \text { LO }^{\#} \\
/ \mu \mathrm{g} \mathrm{L}^{-1}\end{array}$ \\
\hline Dimethyl & 7338 & 130 & 9.8 & 0.9985 & $<0.01$ \\
\hline Benzylbenzoate & 9903 & 145 & 6.5 & 0.9975 & $<0.01$ \\
\hline Diisobutyl & 3606 & 136 & 6.3 & 0.9989 & $<0.01$ \\
\hline Dibutyl & 4150 & 136 & 4.8 & 0.9973 & $<0.01$ \\
\hline Dimethylglycol & 6472 & 137 & 5.7 & 0.9864 & 0.01 \\
\hline Bis(4-methyl-2-pentyl) & 560 & 157 & 9.1 & 0.9866 & 0.01 \\
\hline Bis-ethoxyethyl & 519 & 145 & 10.4 & 0.9985 & 0.01 \\
\hline Bis-pentyl & 7145 & 175 & 7.9 & 0.9908 & 0.01 \\
\hline Hexyl-2-ethylhexyl & 744 & 110 & 3.8 & 0.9988 & 0.01 \\
\hline Benzylbutyl & 457 & 116 & 7.7 & 0.9993 & 0.01 \\
\hline Bis-hexyl & 2675 & 143 & 6.5 & 0.9974 & 0.05 \\
\hline Bis-nbutoxyethyl & 656 & 154 & 9.8 & 0.9981 & 0.05 \\
\hline Biscyclohexyl & 638 & 150 & 10.1 & 0.9990 & 0.05 \\
\hline Bis-2ethylhexyl & 1150 & 126 & 11.3 & 0.9984 & 0.05 \\
\hline Bis-octyl & 931 & 113 & 12.4 & 0.9986 & 0.10 \\
\hline Bis-nonyl & 626 & 167 & 10.8 & 0.9987 & 0.10 \\
\hline
\end{tabular}

* Enrichment ratio without headspace compared to that with headspace.

\# LOD estimated from the $\mathrm{S} / \mathrm{N}$ ratio.

the concentration range of $0.5-50 \mu \mathrm{g} \mathrm{L}^{-1}$. The LOD was estimated from the $\mathrm{S} / \mathrm{N}$ ratio yielding sufficiently low limits of detection (ng $\mathrm{L}^{-1}$ range). Since the CRMs were not available, accuracy validation was not performed. The reported LODs compare to the 3 to $30 \mathrm{ng} \mathrm{L}^{-1}$ reported by Penalver et al. using a $65 \mu \mathrm{m}$ PDMS-DVB SPME with the repeatability and reproducibility in the range of $13-18 \% ;^{38}$ while an order of magnitude lower than of $0.331 \mathrm{ng} \mathrm{L}^{-1}$ to $12.5 \mu \mathrm{g} \mathrm{L} \mathrm{L}^{-1}$ obtained with a headspace sampling using an optical fiber coated with mixed stationary phase of polysiloxane and polymeric fullerene with the ratio of $4: 1{ }^{39}$ The QuEChERS approach yielded significantly higher LODs at between 0.8 and $15 \mu \mathrm{g} \mathrm{kg}^{-1}$ using liquid chromatography and mass spectrometry; ${ }^{40}$ while a magnetic graphene nanocomposite based SPE achieved LODs between 0.01 and $0.04 \mathrm{ng} \mathrm{mL}^{-1}{ }^{41}$ The best comparison is that with the air-assisted liquid-liquid microextraction which achieved the LODs of 0.12 to $1.15 \mathrm{ng} \mathrm{mL} \mathrm{m}^{-1}{ }^{42}$ From the aforegoing comparisons, it is evident that this technique yielded comparative results to those reported in literature; as such it presents a very affordable and viable alternative to the pool of miniaturized extraction techniques for these compounds.
3.8. Application of the Extraction Method Coupled to Hot-water Extraction for the Determination of the Phthalates in a Soil Sample from a Solid-waste Dumpsite

Hot-water extraction has been reported recently for the extraction of herbicides from soil samples. ${ }^{22}$ Owing to its simplicity and efficiency, this approach was tried on the PAE extraction to be coupled with the developed dispersive method. Sadly, none of the analytes was detectable despite some, e.g. bis(2-ethylhexyl) phthalate, being positively detected from the same sample following reflux and Soxhlet extractions using organic solvents in the previous report. ${ }^{17}$ The achieved recoveries on the preextracted soil samples were significantly wider than those obtained from the spiked HPLC grade water ranging from $65-115 \%$ relative to the aqueous standard solutions. This failure could be attributed to the fact that esters hydrolyse easily even in water when subjected to heat, than any other cause such as loss in the method. The same loss in efficiency was reported using sonication-assisted extraction, ${ }^{43}$ where the loss was attributed to the sonication energy enhancing the hydrolysis of these phthalic acid esters. Despite this setback with regards to the soil samples, 
it can still be argued that this method is still applicable for aqueous extractions. Further refinements are necessary to ensure suitability for application towards soil and sediment samples, wherein the detection of the PAEs from the dumpsite can be necessitated.

\section{Conclusions}

The method reported herein adds value to the commonly reported DLLME which uses one dispersing solvent in addition to the extracting solvent resulting in very low solvent recovery after the extraction. This loss of the solvents leads to reduced efficiency since the lost solvent is lost with its pre-extracted analytes. The methods demonstrates sufficient linearity over the concentration range of $0.5-50 \mathrm{ng} \mathrm{mL}^{-1}$ with the coefficient of determination, $R^{2}$ between 0.9866 and 0.9993 . Although the reproducibility was not determined, the method demonstrated sufficient repeatability with the relative standard deviation, $\%$ RSD $\leq 12.5$ under optimized conditions over the entire linearity experiments. The estimated LOD using the $\mathrm{S} / \mathrm{N}$ ratio are sufficiently low for trace analysis $\left(0.01-0.05 \mathrm{ng} \mathrm{mL}^{-1}\right)$ for most of the analytes. The observed values are comparable to those reported in literature for the liquid and solid-based microextractions. However the method yielded very low recoveries when applied to the analysis of spiked soil samples when coupled to hot-water extraction. This poor recovery will be a subject of further studies.

In conclusion, it can be stated that this variation affords a better alternative to the classical dispersive liquid-liquid microextraction with a very minimal solvent loss, while at the same time, it has demonstrated the importance of reduced headspace volume in order to improve the analyte extraction as most of the analytes are lost with the evaporation of the solvent into the headspace volume, thus reducing the extraction efficiency, especially with the addition of salts for increased ionic strength of the solution to effect salting out. These factors add some insights into some of the factors that are commonly ignored in extraction processes.

\section{Acknowledgements}

The authors gratefully acknowledge OPCW (Grant No. L/ICA/ICB/196770/15), National University of Lesotho (Grant No. P116-9008) and University of Botswana for financial support in carrying out this work.

\section{References}

1 Chemical Economics Handbook, Plasticizers. https://www.ihs.com/ products/plasticizers-chemical-economics-handbook.html, 2015 (accessed 15 May 2016).

2 World Health Organisation, Diethylhexyl Phthalate, Environmental Health Criteria, Geneva, 1992, p. 131.

3 S. Net, A. Delmont, R. Sempéré, A. Paluselli and B. Ouddane, Reliable quantification of phthalates in environmental matrices (air, water, sludge, sediment and soil): a review, Sci. Total Environ., 2015, 515, 162-180.

4 W.W. Huber, B. Grasl-Kraupp and R. Schulte-Hermann, Hepatocarcinogenic potential of di (2-ethylhexyl) phthalate in rodents and its implications on human risk, Crit. Rev. Toxicol., 1996, 26(4), 365-481.

5 Center for Drug Evaluation and Research, Guidance for industry limiting the use of certain phthalates as excipients in CDER-regulated products, U.S. Department of Health and Human Services Food and Drug Administration, Washington DC. http://www.fda.gov/Cosmetics/ProductsIngredients/Ingredients/ucm128250.htm, 2012 (accessed 8 June 2016)

6 M. Schade, The right chemistry Home Depot leads chemical clean-up of flooring, GreenBiz, Friday, December 11, 2015, https:/www greenbiz.com/article/home-depot-leads-chemical-cleanup-flooring, 2015 (accessed 8 June 2016).

7 Chemical Watch: Global risk and regulation news, Trend in retailers removing phthalates from flooring continues, Menards joins Lowe's and Home Depot in discontinuing sales, https://chemicalwatch. $\mathrm{com} / 24489$ /trend-in-retailers-removing-phthalates-from-flooringcontinues, 2015 (accessed 8 June 2016)..

8 J.H. Petersen, E.T. Naamansen and P.A. Nielsen, PVC cling film in contact with cheese: health aspects related to global migration and specific migration of DEHA, Food Addit. Contam., 1995, 2, 245-253

9 United States Environmental Protection Agency, National Primary Drinking Water Regulations; Federal Register, Part 12, 40 CFR Part 141, US, Washington DC, USA, July 1st 1991, p. 395.

10 A. Gomez-Hens and M.P. Aguilar-Caballos, Social and economic interest in the control of phthalic acid esters, Trends Anal. Chem., 2003, 22(11), 847-857.

11 M. Cachia, D.W. Southwart and W.H.T. Davison, Identification of mixed plasticizers by a combination of chromatography and infrared spectroscopy, J. Chem. Technol. Biotechnol., 1958, 8(5), 291-293.

12 E. Ayranci and E. Bayram, Adsorption of phthalic acid and its esters onto high-area activated carbon-cloth studied by in situ UV-spectroscopy, J. Hazard. Mater., 2005, 122(1) 147-153.

13 M.C. Zhang, Q.E. Wang and H.S. Zhuang, A novel competitive fluorescence immunoassay for the determination of dibutyl phthalate, Anal. Bioanal. Chem., 2006, 386(5), 1401-1406.

14 A.I. Zia, M.S.A. Rahman, S.C. Mukhopadhyay, P.L. Yu, I.H. Al-Bahadly, C.P. Gooneratne, J. Kosel and T.S. Liao, Technique for rapid detection of phthalates in water and beverages, J. Food Eng., 2013, 116(2), 515-523.

15 Z. Niu, X. Ye, L. Fang, Q. Xue and Z. Sun, Determination of phthalic acid esters in textiles by solid phase extraction-gas chromatography, Se ??pu= Chinese J. Chromatogr./Zhongguo Hua Xue Hui, 2006, 24(5), 503-507.

16 R. Spaccini, X.Y. Song, V. Cozzolino and A. Piccolo, Molecular evaluation of soil organic matter characteristics in three agricultural soils by improved off-line thermochemolysis: the effect of hydrofluoric acid demineralisation treatment, Anal. Chim. Acta, 2013, 802, 46-55.

17 A. Bergé, M. Cladière, J. Gasperi, A. Coursimault, B. Tassin and R. Moilleron, Meta-analysis of environmental contamination by phthalates, Environ. Sci. Pollut. Res., 2013, 20(11), 8057-8076.

18 F. Guo, Q.X. Li and J.P. Alcantara-Licudine, Na4EDTA-assisted sub/supercritical fluid extraction procedure for quantitative recovery of polar analytes in soil, Anal. Chem., 1999, 71(7), 1309-1315.

19 L. Sanchez-Prado, C. Garcia-Jares, T. Dagnac and M. Llompart, Microwave-assisted extraction of emerging pollutants in environmental and biological samples before chromatographic determination, Trends Anal. Chem., 2015, 71, 119-143.

20 M.J. George, Analysis of Endocrine Disrupting Chemicals from the Solid Waste Dumpsite in Maseru using Gas Chromatography and Mass Spectrometry. A report to OPCW, The Hague, Netherlands. 2015.

21 H. Liu and P.K. Dasgupta, Analytical chemistry in a drop. Solvent extraction in a microdrop, Anal. Chem., 1996, 68(11), 1817-1821.

22 M.A. Jeannot and F.F. Cantwell, Solvent microextraction into a single drop, Anal. Chem., 1996, 68(13), 2236-2240.

23 M.A. Farajzadeh, S.M. Sorouraddin and M.R.A. Mogaddam, Liquid phase microextraction of pesticides: a review on current methods, Microchim. Acta, 2014, 181(9-10), 829-851.

24 M.A. Farajzadeh, D. Djozan and P. Khorram, Development of a new microextraction method based on a dynamic single drop in a narrow-bore tube: application in extraction and preconcentration of some organic pollutants in well water and grape juice samples, Talanta, 2011, 85(2), 1135-1142.

25 D.B.G. Williams, M.J. George, R. Meyer and L. Marjanovic, Bubbles in solvent microextraction: the influence of intentionally introduced bubbles on extraction efficiency, Anal. Chem., 2011, 83(17), 6713-6716.

26 M.J. George, L. Marjanovic and D.B.G. Williams, Solvent-assisted headspace sampling using solid phase microextraction for the analysis of phenols in water, Anal. Chem., 2015, 87(19), 9559-9562.

27 T. Letseka and M.J. George, Towards coupling dispersive liquidliquid microextraction with hollow fibre liquid phase microextraction for extraction of organic pollutants of agricultural origin, Anal. Chem. Res., 2016, 10, 28-32.

28 A. Saleh, Y. Yamini, M. Faraji, M. Rezaee and M. Ghambarian, Ultrasound-assisted emulsification microextraction method based on applying low density organic solvents followed by gas chromatography analysis for the determination of polycyclic aromatic hydrocar- 
bons in water samples, J. Chromatogr. A, 2009, 1216(39), 6673-6679.

29 M.J. George, L. Marjanovic and D.B.G. Williams, Picogram-level quantification of some growth hormones in bovine urine using mixed-solvent bubble-in-drop single drop micro-extraction, Talanta, 2015, 144, 445-450.

30 M.J. George, Application of the mixed-solvent BID-SDME technique for determination of some stilbene hormones in water downstream of a cattle slaughterhouse, using gas chromatography and mass spectrometry, Int. J. Environ. Anal. Chem., 2016, 96(3), 247-256.

31 D.B.G. Williams, M.J. George and L. Marjanovic, Rapid detection of atrazine and metolachlor in farm soils: gas chromatography-mass spectrometry-based analysis using the bubble-in-drop single drop microextraction enrichment method, J. Agric. Food Chem., 2014. 62(31),) 7676-7681.

32 M.J. George, Determination and correlation of herbicide residues in water and sediments from the streams flowing into the Caledon River in the eastern Free State using the bubble-in-drop single drop micro-extraction method, Eur. Chem. Bull., 2014,3(10-12), 1098-1102.

33 R.A. Hites, Phthalates in the Charles and the Merrimack rivers, Environ. Health Perspect., 1973, 3, 17-21.

34 P.S. Chen, W.Y. Haung and S.D. Huang, Analysis of triazine herbicides using an up-and-down-shaker-assisted dispersive liquidliquid microextraction coupled with gas chromatography-mass spectrometry, J. Chromatogr. B, 2014, 955, 116-123.

35 M.J. George, Advances in Solvent Microextraction Technique, M.Sc. thesis, University of Johannesburg, South Africa, 2007.

36 K. Hori, Y. Ikenaga, K. Arata, T. Takahashi, K. Kasai, Y. Noguchi, M. Sumimoto and $\mathrm{H}$. Yamamoto, Theoretical study on the reaction mechanism for the hydrolysis of esters and amides under acidic conditions, Tetrahedron, 2007, 63(5), 1264-1269.

37 V. Theodorou, K. Skobridis, A.G. Tzakos and V. Ragoussis, A simple method for the alkaline hydrolysis of esters, Tetrahedron Lett., 2007, 48(46) 8230-8233.

38 A. Penalver, E. Pocurull, F. Borrull and R.M. Marcé, Comparison of different fibers for the solid-phase microextraction of phthalate esters from water, J. Chromatogr. A, 2001, 922(1), 377-384.

39 Z.L. Liu, C.H. Xiao, C.Y. Wu and H.M. Han, Determination of phthalic diesters in water by solid phase microextraction-capillary gas chromatography, Se ??pu= Chinese J. Chromatogr./Zhongguo Hua Xue Hui, 2000, 18, 568-570

40 D. Xu, X. Deng, E. Fang, X. Zheng, Y. Zhou, L. Lin, L. Chen, H. Wu and Z. Huang, Determination of 23 phthalic acid esters in food by liquid chromatography tandem mass spectrometry, J. Chromatogr. A, 2014, 1324, 49-56.

41 Q. Wu, M. Liu, X. Ma, W. Wang, C. Wang, X. Zang and Z. Wang, Extraction of phthalate esters from water and beverages using a graphene-based magnetic nanocomposite prior to their determination by HPLC, Microchim. Acta, 2012, 177(1-2), 23-30.

42 M.A. Farajzadeh and M.R. Mogaddam, Air-assisted liquid-liquid microextraction method as a novel microextraction technique; application in extraction and preconcentration of phthalate esters in aqueous sample followed by gas chromatography-flame ionization detection, Anal. Chim. Acta, 2012,728, 31-38.

43 B. Yim, Y. Nagata and Y. Maeda, Sonolytic degradation of phthalic acid esters in aqueous solutions. Acceleration of hydrolysis by sonochemical action, J. Phys. Chem. A, 2002, 106(1), 104-107. 